\title{
Global Optimisation of Hydroxylated Silica Clusters: a Cascade Monte Carlo Basin Hopping Approach
}

\author{
Andi Cuko ${ }^{1,2}$, Toni Maciá ${ }^{1}$, Monica Calatayud ${ }^{2,3}$, Stefan T. Bromley ${ }^{1,4^{*}}$ \\ ${ }^{1}$ Departament de Química Física and Institut de Química Teòrica i Computacional (IQTCUB), \\ Universitat de Barcelona, E-08028 Barcelona, Spain \\ ${ }^{2}$ SorbonneUniversités, UPMC Univ Paris 06, CNRS, Laboratoire de Chimie Théorique CC 137 - \\ 4, place Jussieu F. 75252 PARIS CEDEX 05 - France \\ ${ }^{3}$ Institut Universitaire de France, France \\ ${ }^{4}$ Institució Catalana de Recerca i Estudis Avançats (ICREA), E-08010 Barcelona, Spain \\ *Corresponding author: s.bromley@ub.edu
}

\begin{abstract}
We report on a global optimisation study of hydroxylated silica nanoclusters $\left(\mathrm{SiO}_{2}\right)_{M} \cdot\left(\mathrm{H}_{2} \mathrm{O}\right)_{N}$ with sizes $M=6,8,1012$, and for each size with a variable number of incorporated water molecules $(N=1,2$, 3...). Due to the high structural complexity of these systems and the associated ruggedness of the underlying potential energy landscape, we propose a "cascade" global optimisation approach. Specifically, we use Monte Carlo Basin Hopping (MCBH) where for each step we employ two energy minimisations with: (i) a few-term simple but computationally efficient interatomic potential (IP) which does not distinguish between $\mathrm{H}$-bonded conformational isomers, and then (ii) a more sophisticated IP which accounts for polarisation and $\mathrm{H}$-bonding. Final energies from the MCBH search are then refined with optimisations using density functional theory. The reliability of our approach is first established via comparison with previously reported results for the $\left(\mathrm{SiO}_{2}\right)_{8} \cdot\left(\mathrm{H}_{2} \mathrm{O}\right)_{N}$ case, and then applied to the $M=6,10$ and 12 systems. For all systems studied our results follow the trend in hydroxylation energy versus $\mathrm{N}$, whereby the energy gain with hydroxylation is found to level off at a point where the average tetrahedral distortion of the $\mathrm{SiO}_{4}$ centres is minimised. This optimal hydroxylation point is further found to follow an inverse power law with increasing cluster size $(M)$ with an exponent close to $-2 / 3$, further confirming work in previous studies for other cluster sizes.
\end{abstract}




\section{Introduction}

Nanosized silica particles are widely used during the preparation of materials (e.g. cement-based materials,[1] coatings,[2] polymers[3]) to significantly enhance their physical and mechanical properties. In addition to their high intrinsic strength (e.g. the $\mathrm{Si}-\mathrm{O}$ bond $30 \%$ being stronger than the C-C bond), the success of silica nanoparticles for such applications is also due them having a relatively high proportion of reactive surface groups which can strongly interact with host materials. Silica is particularly prone to reaction with water, commonly resulting in a surface coverage of hydroxyl (Si-OH) groups.[4] The degree of hydroxylation is found to determine many of the properties of nanosilica. In solution, for example, the $\mathrm{pH}$ determines the solubility/hydroxylation of small silicate nanoparticles. Such species are central to the synthesis of technologically important nanoporous silicate materials such as zeolites[5] and occur in (bio)mineral nucleation, growth and dissolution processes.[6] The proportion of hydroxyls on small silica particles can also be deliberately increasing by physico-chemical processing to produce enhanced properties for applications (e.g. epoxidation catalysis[7], mechanically robust antireflective films[8]). The density of surface hydroxyls has further been reported to influence the biocompatibility and toxicity of silica nanoparticles. $[9,10]$

From a computational perspective the reaction of water with silica surfaces has attracted much attention.[11] For atomic to nanosized hydroxylated silica systems studies have tended to either focus on the detailed thermodynamics of the condensation of small ring- or chain-like $\left(\mathrm{SiO}_{2}\right)_{M}$ $N \leq 6$ oligomers in solution[12,13] using ab initio methods, or on larger scale (i.e. with $100 \mathrm{~s}$ or $1000 \mathrm{~s}$ of atoms) classical forcefield simulations of hydroxylated nanosilca.[14,15,16] Between these extremes, some recent studies have focussed on attempting to find the most stable structures of hydroxylated silica nanoclusters in the approximate size range of $10-100$ atoms.[17,18,19,20] This intermediate size regime is characterised by having non-trivial (i.e. not simply rings or chains) structures which are also not totally amorphous as typically assumed for larger nanoparticles. Hydroxylated silica nanoparticles with such sizes can thus be thought of as species which represent a structurally complex molecular-bulk crossover regime. Such species are likely to be particularly important in understanding nucleation of complex silica solids (e.g. zeolites). Although in such processes, many factors play an important role (e.g. $\mathrm{pH}$, templates)[5] knowing the lowest energy hydroxylated nanosilica species in the absence of such factors provides a baseline to assess how and when they are important. Previous theoretical work in this size regime has specifically investigated hydroxylated silica clusters $\left(\mathrm{SiO}_{2}\right)_{M} \cdot\left(\mathrm{H}_{2} \mathrm{O}\right)_{N}$ with sizes $M=4,8,16,24$, and each with a variable number of incorporated water molecules $(N=1,2,3 \ldots)[17,18,19,20]$ Due to their high structural complexity, global optimisation was employed in these studies to find low energy isomers for each stoichiometry. Specifically, a fairly simple set of interatomic potentials was used in conjunction with the Monte Carlo basin hopping (MCBH)[21] approach to first obtain initial cluster structures. Due to approximations in the IP set employed these non-refined structures provide only a poor account of $\mathrm{OH} \cdots \mathrm{OH}$ hydrogen bonding on the surfaces of the hydroxylated nanoclusters.[19] Optimisations using relatively computationally expensive density functional theory (DFT) calculations on a selection of IP-MCBH-generated structures were used to provide final clusters exhibiting hydrogen bonding.

In this work we report on a MCBH global optimization approach to hydroxylated silica nanosystems employing a cascade local optimization method. First, our method is shown to be reliable by applying it to the previously studied $\left(\mathrm{SiO}_{2}\right)_{8} \cdot\left(\mathrm{H}_{2} \mathrm{O}\right)_{N}$ system. $[18,19]$ We then, generate global minima candidates for the as yet unreported systems: $\left(\mathrm{SiO}_{2}\right)_{M} \cdot\left(\mathrm{H}_{2} \mathrm{O}\right)_{N}, M=6,10$, 12, each for a 
range of $N$ values. In previous work it was shown that the hydroxylation reaction energy for silica clusters decreases by addition water molecules until it levels off around an optimal number $N$. This optimal number was found to be cluster size dependent to follow an inverse power law of the form $2 M^{-2 / 3}$. Using our new global minima candidates for $\left(\mathrm{SiO}_{2}\right)_{M} \cdot\left(\mathrm{H}_{2} \mathrm{O}\right)_{N}, \mathrm{M}=6,10,12$ we also further probe the generality of this relation.

\section{Methodology}

In this work we apply the $\mathrm{MCBH}$ global optimization technique to find low energy global minima candidates for the cluster set $\left(\mathrm{SiO}_{2}\right)_{M} \cdot\left(\mathrm{H}_{2} \mathrm{O}\right)_{N}, M=6,8,10,12$ for a range of $N$. The general idea behind this technique in based on repeated steps consisting of random structural distortion and a subsequent local optimization. The Metropolis criterion[22] is applied in order to accept or refuse the local optimized structure according to a cost in passing from the initial (before the random distortion) and the final state, and a fictitious temperature. Usually the cost is the energy difference between the two states but in principle it is possible to generate other cost criteria based on system properties. Within a certain (unknown) number of the cyclic repetitions of this procedure is possible to explore the potential energy surface (PES) of the system. At each step, the structure can be locally optimized with both $a b$ initio or a classical IP. In the first case, the applicability is limited to relatively small systems due to the high computational expense. This expense not only originates from local optimizations of the system but also from the higher number of steps required to properly sample the PES. Indeed, in principle, the number of energy minima is exponentially proportional to the number of atoms. A typical approach to makes MCBH calculations more computationally amenable is to use IPs for efficient optimisation. Nevertheless, IPs alone, often, cannot be relied upon for an accurate description of a system's properties. Therefore, after a an IP-based MCBH run, a certain number of resulting low energy structures can be re-optimized with more accurate methods (e.g. DFT). For some systems, quite accurate IPs have been developed for which parameters have been fitted to experimental and/or ab initio data. In principle, the better the IP, the lower the number of structures that require significant further optimization with a more accurate method. However, we note that to have an IP with higher accuracy, you typically require correspondingly more complex functional forms with a higher number of terms. In turn, during a MCBH run, the direct use of such IPs can lead to practical problems in relaxing structures far from the equilibrium geometry (after a random distortion). Here we present a $\mathrm{MCBH}$ procedure in which for each step, a cascade structure optimization is performed with two different IPs. The general idea is to first use a robust and efficient IP to pre-optimize a distorted structure and then use more accurate IP for fully relaxing this pre-optimized structure. The Metropolis criterion is than applied after the second optimization. Our cascade-MCBH approach interfaces with the GULP package[23] which offers the possibility of using many IP types. We have implemented this procedure using Atomic Simulation Environment[24] (ASE) modules together.

In the literature, IPs for bare silica $\left(\mathrm{SiO}_{2}\right)$ bulk systems, such as TTAM[25] and BKS, [26] have been successfully used. Hassanali and Singer (HS)[27] introduced a IP parameterization for the silicawater interface by adding new three-body terms terms to the BKS IP in order to describe Si-O-H and $\mathrm{H}$-bonds. All these IPs are based on the Buckingham mathematical form as shown in equation (1) 
$V_{i j}\left(r_{i j}\right)=\frac{q_{i} q_{j}}{4 \pi \varepsilon_{0} r_{i j}}+A_{i j} \exp \left(-\frac{r_{i j}}{B_{i j}}\right)-\frac{C_{i j}}{r_{i j}^{6}}$

where $\mathrm{i}, \mathrm{j} \in\{\mathrm{Si}, \mathrm{O}\}, \mathrm{r}_{\mathrm{ij}}$ is the interionic separation, $\mathrm{q}_{\mathrm{i}, \mathrm{j}}$ are the ionic charges and $\mathrm{A}, \mathrm{B}$ and $\mathrm{C}$ are fitted parameters. With respect to nanosilica, Flikkema and Bromley (FB) re-parameterised a Buckingham IP specifically for treating bare silica nanoclusters.[28] Recently, Pedone et al.[29] have parameterized a new and more complex Buckingham IP with polarisable ions for hydroxylated silica. This IP also incorporates intra- and inter-bond Morse potentials and a three-body term for hydrogen bonds was shown to provide good structures and vibrational frequencies with respect to experimental and DFT-calculated data on hydroxylated silica surfaces. Recently, a systematic study has showed that this IP is also accurate for the treatment of hydroxylated nanoparticles.[30]

In previous global optimization studies the FB IP and a simplified version of the HS IP ( $\left.\mathrm{HS}_{\text {simp }}\right)$ has been used for finding low energy clusters where the HS three-body terms were omitted for simplicity and computational efficiency.[17,18] By omitting these terms, the correct description of hydroxyl groups $(\mathrm{OH})$ is lost, therefore, the clusters do not have any $\mathrm{H}$-bond contribution to their stability (see Fig. 1). In this way, isomers stabilized by hydrogen bonds contributions are not easily found only using $\mathrm{HS}_{\text {simp }}$ in a $\mathrm{MCBH}$ run. Such concerns are especially relevant to relatively large clusters and/or a high hydroxylation degree where $\mathrm{H}$-bonding can influence the energetic ordering and structure of low lying minima (each $\mathrm{H}$-bond contributes of about $20 \mathrm{~kJ} / \mathrm{mol}$ ). Thus for silica nanoparticles with increasing size and/or with moderate to high hydroxylation a good description of $\mathrm{OH}$ groups and their $\mathrm{H}$-bonding becomes essential in order to avoid missing possible low energy clusters.

In this work we consider hydroxylated silica clusters $\left(\mathrm{SiO}_{2}\right)_{M} \cdot\left(\mathrm{H}_{2} \mathrm{O}\right)_{N}$ systems with $M=6,810$ and 12 each one with different hydroxylation ratio $N: M\left(R_{N / M}\right)$ up to $\sim 65 \%$. As a pre-optimization in the first cascade step we use the FB IP with $\mathrm{OH}$ groups described by $\mathrm{HS}_{\text {simp }}$ IP (IP_1), while for second part of the cascade we use the IP due to Pedone et al. (IP_2)[29]. In general, the mathematical form of a Buckingham IP energy entails that the energy of a pair of atoms tends to - $\infty$ when their distance tends to 0 . To avoid such a superposition of atoms caused by random displacements during a MCBH run we added very short range repulsive terms to the Buckingham describing the interaction between unlike charged species in IP_1. In order to more efficiently explore the PES we use a selfcorrecting temperature technique to keep the accept ratio between 60 and $80 \%$. For each MCBH step atomic displacements are set to a maximum of $\pm 0.8 \AA$ for any cartesian coordinate and the energy difference used to discriminate between two different structures is set to $1 \mathrm{~kJ} / \mathrm{mol}$. Several runs (at least three) of fifty thousand steps each are performed for all considered systems. The ten most energetically stable isomers resulting from the MCBH global optimization procedure for each system stoichiometry are subsequently refined using DFT. These final optimisations are done employing the FHI-AIMS package[31], using the B3LYP[32] hybrid functional and a tier-1 atomic centred numerical basis set with a light integration grid with. The B3LYP functional has been shown to well describe hydroxylated silica in numerous previous studies.[17,19,33,34] 
Global optimization
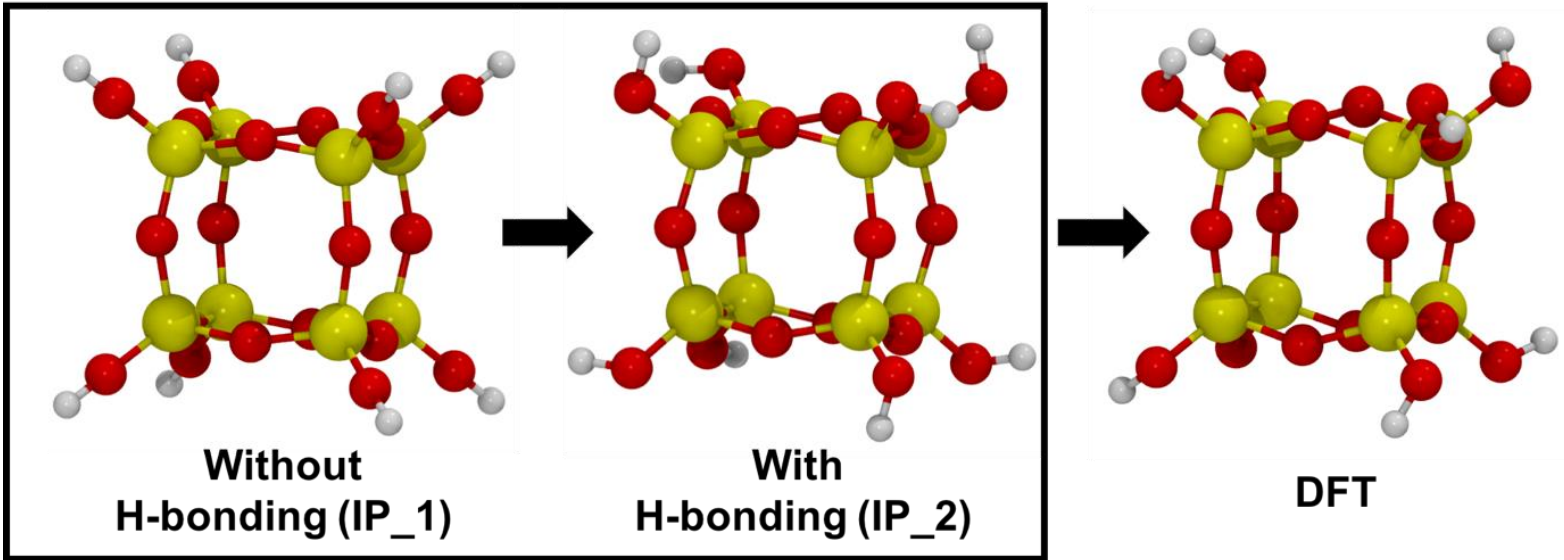

Fig. 1 Structures of a $\left(\mathrm{SiO}_{2}\right)_{8} \cdot\left(\mathrm{H}_{2} \mathrm{O}\right)_{4}$ nanocluster optimized with: IP_1 used in the first cascade step (left), IP_2 used in the second cascade step (middle), and DFT using B3LYP (right). Atom colour key: Si - yellow, Si, O - red, H - grey.

\section{Results and Discussions}

We first tested our cascade MCBH methodology on $\left(\mathrm{SiO}_{2}\right)_{8} \cdot\left(\mathrm{H}_{2} \mathrm{O}\right)_{N}$ systems with $N=2-5$ incorporated water molecules as previously studied in refs [18] and [19]. Specifically, we consider the three most stable isomers we find for each series with those previously reported. In Fig. 2 we report isomers structures found both from this work and from refs [18] and [19].

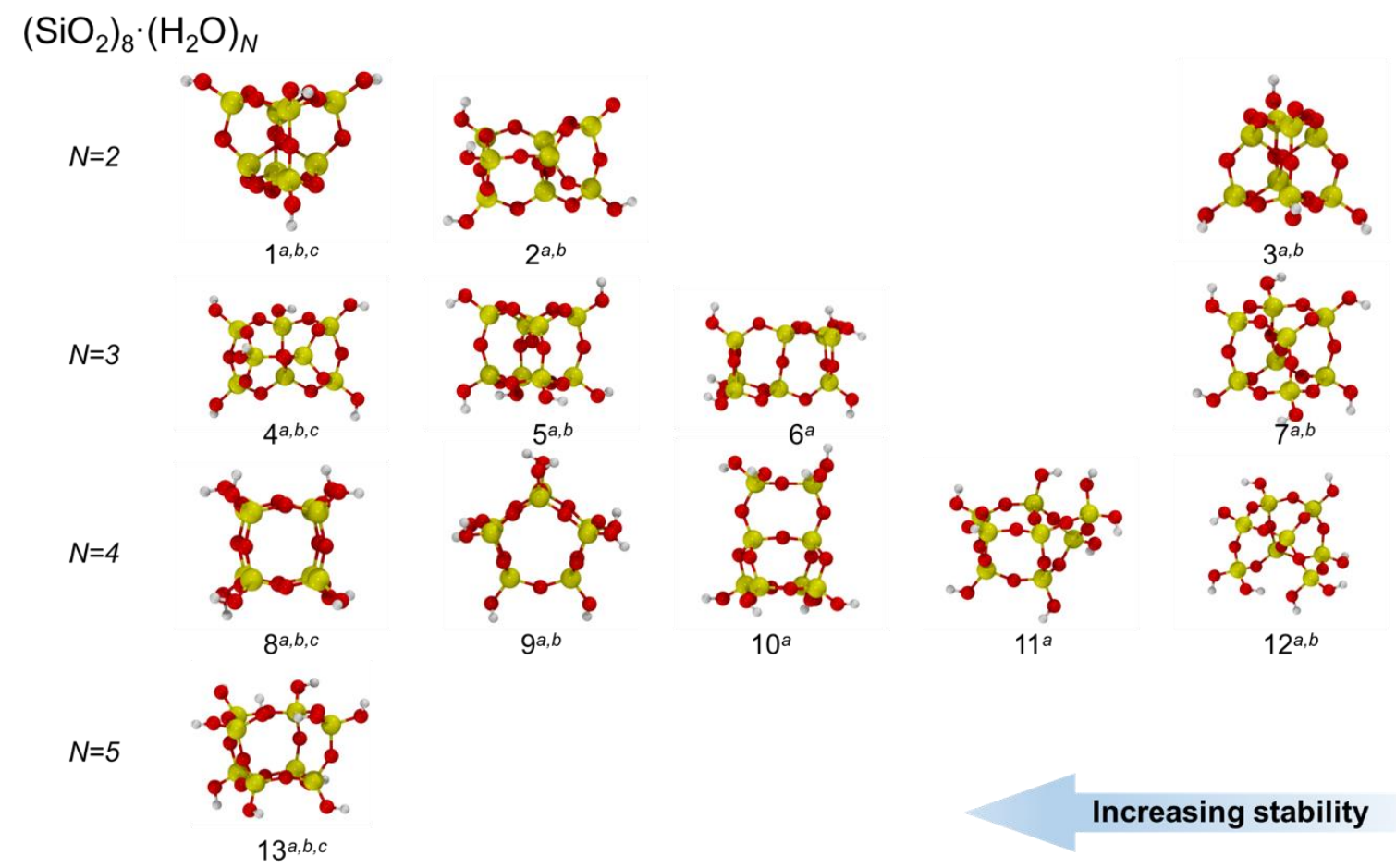

Fig. 2 Structures of $\left(\mathrm{SiO}_{2}\right)_{8} \cdot\left(\mathrm{H}_{2} \mathrm{O}\right)_{N}$ nanoclusters obtained in this work $\left({ }^{a}\right)$, ref [19] as $\left({ }^{b}\right)$ and in ref [18] as $\left({ }^{c}\right)$. Clusters for each $N$ are ordered from left to right according to decreasing energetic stability. In parentheses energy differeneces with respect to the best global minimum candidate (far left of each row) are reported in kj/mol. Atom colour key: $\mathrm{Si}-$ yellow, $\mathrm{Si}, \mathrm{O}-\mathrm{red}$, $\mathrm{H}$ - grey. 
In our comparison we take all stable conformational isomers related to different $\mathrm{H}$-bonding patterns of the hydroxyls on a single Si-O skeleton with a fixed connectivity to be representations of the same isomer. Typically, the total energy range due to different $\mathrm{H}$-bonding patterns on the same isomer of this size is $1-10 \mathrm{~kJ} / \mathrm{mol}$. In all cases we report isomers with the lowest energy $\mathrm{H}$-bonded pattern found. We note that although the first part of the cascade does not provide a correct description of $\mathrm{H}$-bonding, in the second part of the cascade several $\mathrm{H}$-bond patterns are sampled which ensures that the conformational space hydroxyl orientations is well explored. This part of the cascade is particularly important for relatively large and highly hydroxylated clusters where $\mathrm{H}$-bonding can contribute a relatively large amount to total energy differences.

In the case of the most energetically stable structures for each $\mathrm{N}$ considered (i.e. Fig. 2, isomers 1, 4, 8 and 13) we found the same isomers as in previous works. This result further confirms these candidate structures as global minima. The second most stable isomers for each $\mathrm{N}$ (i.e. Fig. 2, isomers 2, 5 and 10) also confirm the corresponding results of refs [18] and [19]. For the third most stable isomers, for two incorporated water molecules $(N=2)$ we also found the previously reported isomer structures. Using the cascade method, however, we were able to find more energetically stable candidates for $N=3$ (Fig. 2, isomer 6 being $3 \mathrm{~kJ} / \mathrm{mol}$ lower than cluster 7) and $N=4$ (isomers 14 and $22 \mathrm{~kJ} / \mathrm{mol}$ lower than cluster 12). For $N=5$ we confirm the global minimum candidate isomer structure and other low energy isomers are reported in literature.

After clearly demonstrating the capacity of our cascade MCBH approach for hydroxylated silica clusters for $M=8$, we applied our method for to the, as yet unreported, systems: $\left(\mathrm{SiO}_{2}\right)_{M^{\prime}} \cdot\left(\mathrm{H}_{2} \mathrm{O}\right)_{N}$ with $M=6,10,12$, each for a range of $\mathrm{N}$. The obtained new global minima candidate clusters are shown in figure 3 . Low numbers of incorporated water (i.e. $N=1,2$ ) do not fully hydroxylate the one-coordinated defective oxygen centres present in anhydrous silica clusters. There are two types of singly-coordinated dangling oxygen defects: (i) oxygen atoms in silanone species (formally: $>\mathrm{Si}=\mathrm{O}$, but arguably more accurately: $>\mathrm{Si}^{+}-\mathrm{O}^{-}[35,36]$ ) having three-coordinated $\mathrm{Si}-$ centres, and (ii) non-bridging oxygen (NBO) atoms accompanied with compensating triple coordinated oxygen-sites, also known a compensated NBOs or valence alternation pairs [37,38]. These defective centres are well studied in literature and it is known that they can readily be hydroxylated through reaction with water $[4,11]$. By increasing the number of incorporated water on an already fully hydroxylated cluster, bridging oxygens (Si-O-Si) are broken and the formation of silanols $(\mathrm{Si}-\mathrm{OH})$ occur until there is one $-\mathrm{OH}$ group for each $\mathrm{Si}$-centre. Further hydroxylation tends to lead to the formation of geminal silanols (i.e. two $\mathrm{OH}$ groups per $\mathrm{Si}$ ). The hydroxylation reaction $\left(\Delta E_{\text {hyx }}\right)$ energy:

$\Delta E_{h y x}=E_{\left(\mathrm{SiO}_{2}\right)_{M}\left(\mathrm{H}_{2} \mathrm{O}\right)_{N}}-\left(E_{\left(\mathrm{SiO}_{2}\right)_{M}}+N E_{\left(\mathrm{H}_{2} \mathrm{O}\right)}\right)$

indicates the relative stability of the hydroxylated structures with $N$ incorporated water molecules with respect to the equivalent anhydrous silica system. This quantity indicates the tendency of anhydrous silica to be hydroxylated, in particular if $\Delta E_{h y x}$ is negative the reaction is energetically favourable. Although strictly speaking a thermodynamically favourable reaction is evaluated by the free energy difference, we neglect the entropic contributions and assume that $\Delta E_{h y x}$ a good indicator of reaction favourability. 


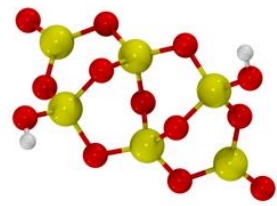

1: $M=6, N=1$

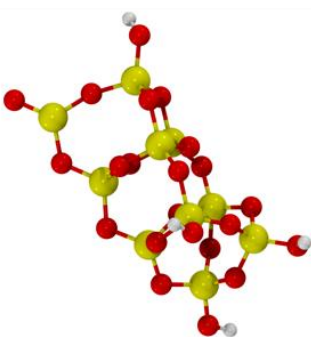

5: $M=10, N=2$

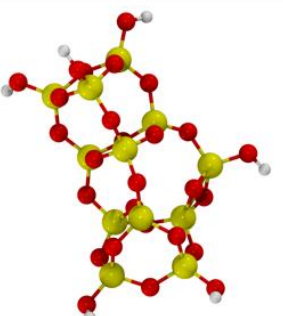

9: $M=12, N=3$

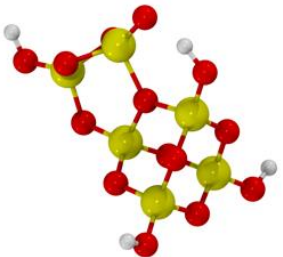

2: $M=6, N=2$

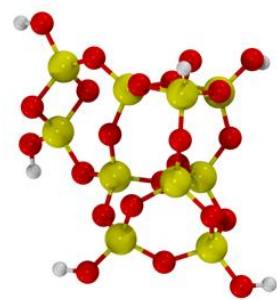

6: $M=10, N=3$

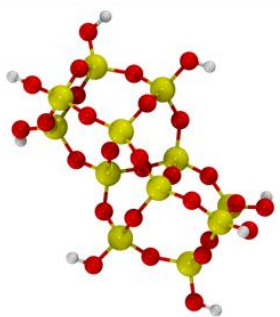

10: $M=12, N=4$

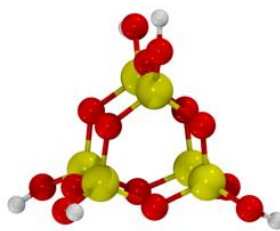

3: $M=6, N=3$

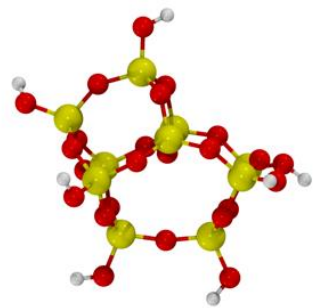

7: $M=10, N=4$

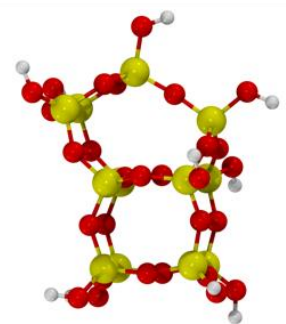

11: $M=12, N=5$

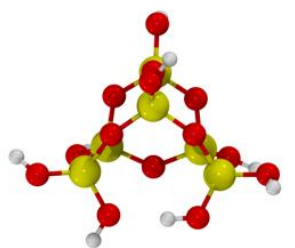

4: $M=6, N=4$

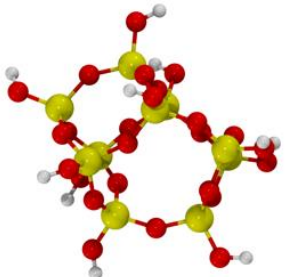

8: $M=10, N=5$

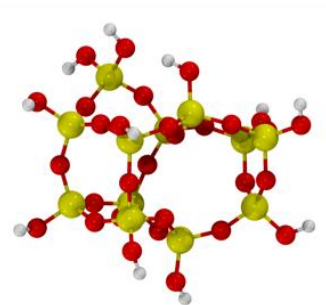

12: $M=12, N=6$

Fig. 3 The most energetically stable $\left(\mathrm{SiO}_{2}\right)_{M} \cdot\left(\mathrm{H}_{2} \mathrm{O}\right)_{N}$ nanoclusters obtained in this work for $M=6,10$ and 12 silica units, each with a range of incorporated water molecule $(N)$ corresponding to a degree of hydroxylation $\left(R_{N / M}=N / M\right)$ between 17 $67 \%$. Atom colour key: $\mathrm{Si}-$ yellow, $\mathrm{Si}, \mathrm{O}-$ red, $\mathrm{H}$ - grey. 

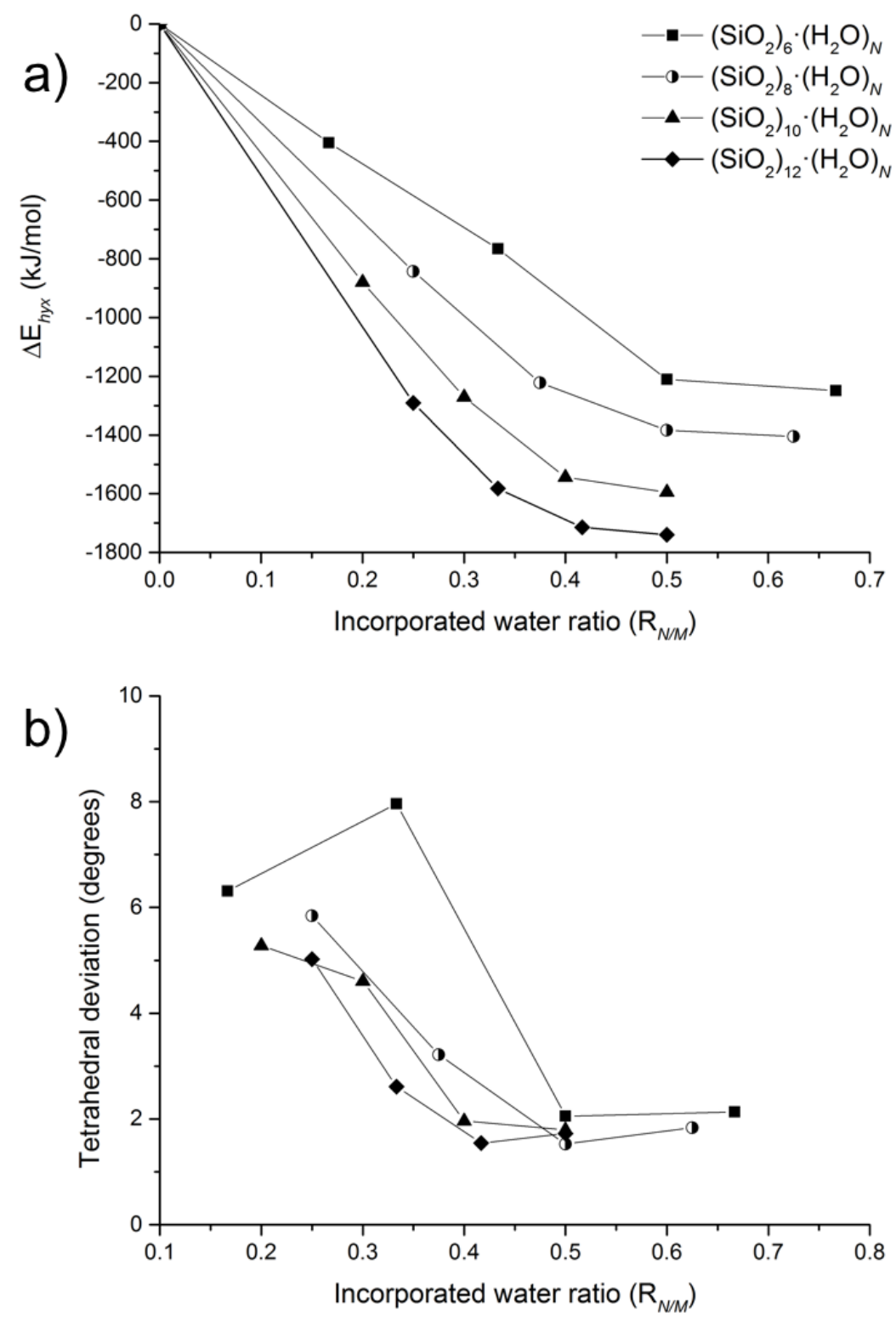

Fig. 4 a) Hydroxylation delta energy $\left(\Delta \mathrm{E}_{\text {hyx }}\right)$ in $\mathrm{kJ} / \mathrm{mol}$ relative to the correspondingly sized global minima $\left(\mathrm{SiO}_{2}\right)_{M} \cdot\left(\mathrm{H}_{2} \mathrm{O}\right)_{N}$ clusters for $M=6,8,10,12$ with respect to incorporated water ratio $\left(\mathrm{R}_{N / M}\right)$. b) Deviations from the ideal silicon-centred tetrahedron $\left(\mathrm{SiO}_{4}\right)$ in degrees with respect to hydroxylation degree. Symbols for $M=8$ shown as half filled circles are also reported in ref [17] and reproduced by us in this work.

In figure 4 we show hydroxylation energies (Fig. 4a) and $\mathrm{Si}$-centred $\mathrm{SiO}_{4}$ tetrahedral distortions (Fig. $4 b)$ with respect to incorporated water ratio $\left(R_{N / M}=N / M\right)$ for the most stable $\left(\mathrm{SiO}_{2}\right)_{M} \cdot\left(\mathrm{H}_{2} \mathrm{O}\right)_{N}$ candidates with $M=6,8,10,12$. The tetrahedral distortion is taken as the average of the differences between the root mean square of the six O-Si-O angles relative to optimal unstrained value of 109.47 for each $\mathrm{SiO}_{4}$ centre. Generally, structures made mainly with rings containing more than three -(Si-O)- units are found to have low tetrahedral distortion.[39] Interestingly, several of the structures shown in Fig. 3, can be viewed as being generated from condensation of building blocks of smaller sized isomers. The most common building blocks observed, for instance, are the $\left(\mathrm{SiO}_{2}\right)_{6} \cdot\left(\mathrm{H}_{2} \mathrm{O}\right)_{3}$ trigonal prism (Fig. 2: isomer 3), the $\left(\mathrm{SiO}_{2}\right)_{8} \cdot\left(\mathrm{H}_{2} \mathrm{O}\right)_{4}$ cubic structure (Fig. 1: isomer 8 - 
also known as a double 4-ring or D4R) and the pentagonal $\left(\mathrm{SiO}_{2}\right)_{8} \cdot\left(\mathrm{H}_{2} \mathrm{O}\right)_{4}$ structure (Fig. 1: isomer 9). As the cluster size increase the most common building block becomes the D4R (e.g. see isomers 9 and 11 in Fig. 3) which only exhibits rings with low tetrahedral distortion. Larger isomers in other work also commonly exhibit the cubic $\left(\mathrm{SiO}_{2}\right)_{8^{\cdot}} \cdot\left(\mathrm{H}_{2} \mathrm{O}\right)_{4}$ D4R unit (e.g. $\left(\mathrm{SiO}_{2}\right)_{24} \cdot\left(\mathrm{H}_{2} \mathrm{O}\right)_{12}$ in ref [18] which can be considered as a condensation of $\mathrm{D} 4 \mathrm{R}$ units in triangular arrangement).

$\Delta E_{\text {hyx }}$ is found to be negative and monotonically decreasing with respect to the number of incorporated water molecules showing that the hydroxylation reaction of anhydrous silica is always energetically favoured. We also notice an energetic down shift with respect to $M$ for the different $N$ series, due to the increasing of the size of the system. The decreasing of $\Delta E_{h y x}$ in a series of $N$ values for a fixed size $M$, can be split into the contribution by three factors: i) at a low number of incorporated water molecules, the gain in energy is mainly due to hydroxylation of the defective oxygen centres, ii) once a cluster is fully hydroxylated (defective centres are no longer present), $\Delta E_{\text {hyx }}$ drops because adding further water molecules helps to decrease the tetrahedral distortion of Si-centres (e.g. by hydrolysing small strained rings), iii) at a high number of incorporated water molecules there is a competition between two effects affecting $\Delta E_{\text {hyx }}$ : the formation of geminal silanols (energetically not favoured effect since $\mathrm{O}-\mathrm{Si}$ is stronger a bond than $\mathrm{O}-\mathrm{H}$ ), and the formation of hydrogen bonds which is an energetically stabilizing effect. These latter two contributions in phase (iii) seem to be almost equivalent energetically, leading to the drop in $\Delta E_{\text {hyx }}$ tending to level off. The hydroxylation degree at the transition point between the ii) and the iii) phases can be regarded as the optimal hydroxylation degree $\left(R_{N / M}^{o p t}\right)$. In other words, $\left(R_{N / M}^{o p t}\right)$ is the $\mathrm{R}_{N / M}$ value where the hydroxylation reaction energy first levels off to a flatter linear regime due to the minimum tetrahedral distortion being reached with increasing hydroxylation.

$R_{N / M}^{o p t}$ was first introduced in ref [18] for systems with $M=4,8,16,24$. Specifically, $R_{N / M}^{o p t}$ was tracked with respect to the number of $\mathrm{SiO}_{2}$ units $(M)$ in order to examine its size dependency. Herein we further add $R_{N / M}^{o p t}$ values for the cluster sizes $M=6,10,12$ silica sizes. To extract $R_{N / M}^{o p t}$ values for each $\left(\mathrm{SiO}_{2}\right)_{M}$ series we choose the $\mathrm{R}_{N / M}$ value that correspond to the cluster with the lowest tetrahedral distortion which is consistent with choice made in ref [18]. The selected $R_{N / M}^{o p t}$ values for clusters with $M=6,10,12$ correspond respectively to structures $3,8,11$ in figure 3 . Although the choice of $R_{N / M}^{o p t}=0.5$ for the set of $\left(\mathrm{SiO}_{2}\right)_{10} \cdot\left(\mathrm{H}_{2} \mathrm{O}\right)_{N}$ clusters is consistent with the $\mathrm{R}_{N / M}$ value that lowers the tetrahedral distortion most, we notice that the $R_{N / M}$ value where tetrahedral distortion levels off (i.e. 0.4) would be likewise acceptable. 


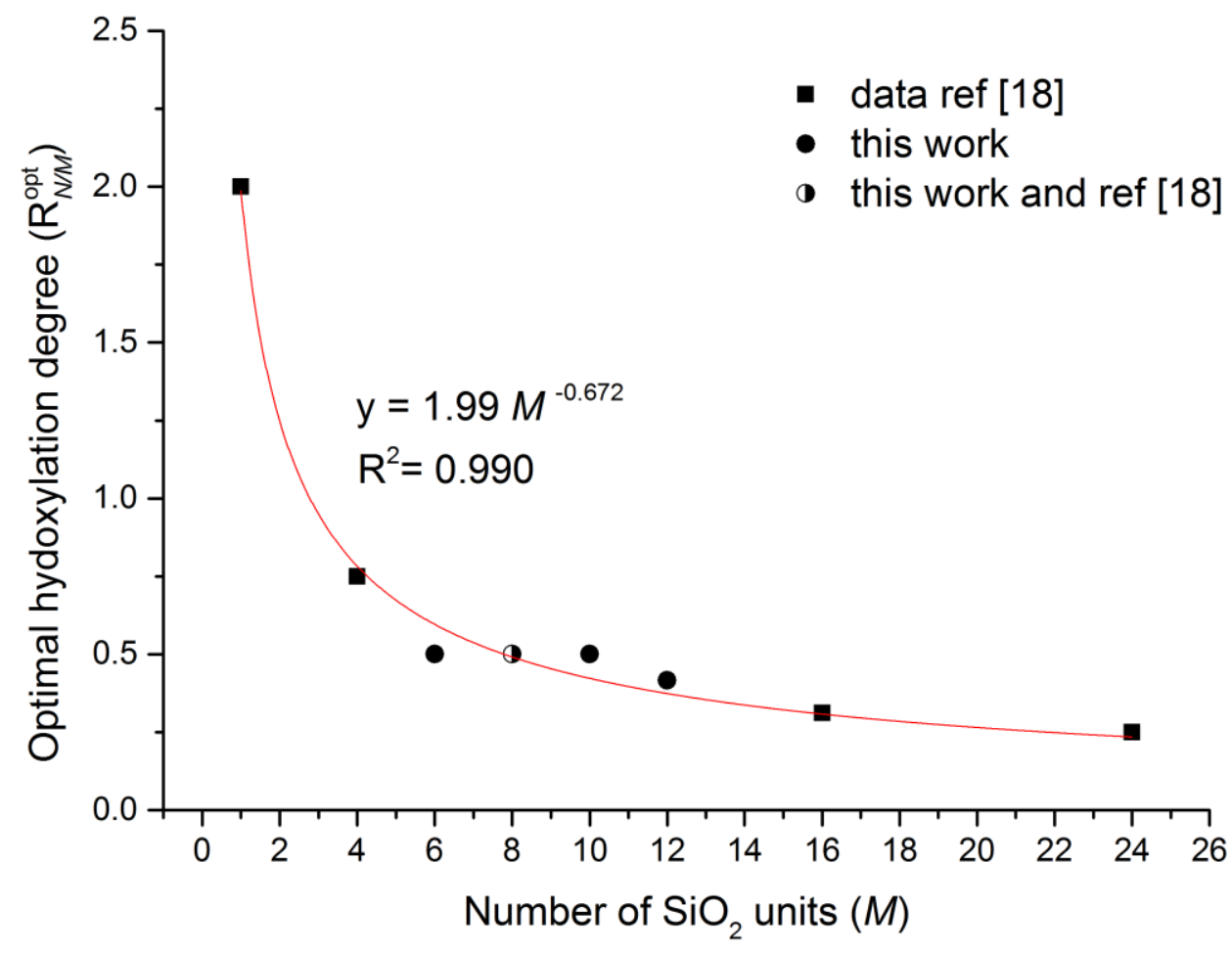

Fig. 5 Data for optimal hydroxylation degree $\left(\mathrm{R}_{N / M}^{\text {opt }}\right)$ with respect to cluster size fitted with an inverse power law. Black filled square symbols correspond to data from ref [18].

$R_{N / M}^{o p t}$ with respect to the number of $\mathrm{SiO}_{2}$ units $(M)$ is shown in Fig. 5 with data from ref [18] in black filled squares and data from this work in black filled circles. The half-filled circle correspond to the $\left(\mathrm{SiO}_{2}\right)_{8} \cdot\left(\mathrm{H}_{2} \mathrm{O}\right)_{N}$ set both studied previously and used in this work as test case. The first point of the series $(M=1)$ corresponds orthosilicic acid $\left(\mathrm{Si}(\mathrm{OH})_{4}\right)$ where $R_{N / M}^{o p t}=2 . R_{N / M}^{o p t}$ is higher for smaller clusters and drop as the size increases and tends to follow an inverse power law. The new data generated in this work $(M=6,10,12)$ do not bring to significant changes in the power law equation in ref [18] and confirm the $R_{N / M}^{o p t}$ size dependency trend. Interestingly, the exponent of the inverse power law is close to $-2 / 3$ while the general size dependency of many properties of a generic nanoparticles scale with a inverse power law but with an exponent of -1/3.[40] Generally, the $-1 / 3$ exponent derives from the surface-to-volume ration assuming a spherical nanoparticle shape. For a generic property size dependency, we expect a small deviation in the exponent from $-1 / 3$ due nonspherical shape of real structures and possibility due to property fluctuations for their extreme dependency on small changes when systems are relatively small. Nevertheless, the $-2 / 3$ exponent in $R_{N / M}^{o p t}$ size dependency seems to be affected by some unknown factors for such deviation from $-1 / 3$ that we hope to elucidate in future works.

\section{Conclusions}

In this work we present a methodology for performing IP-DFT basing hopping global optimization studies on systems such as hydroxylated silica clusters where using an accurate IPs is essential to 
correctly describe hydrogen bonds. For the $\left(\mathrm{SiO}_{2}\right)_{8} \cdot\left(\mathrm{H}_{2} \mathrm{O}\right)_{N}$ test case, the cascade approach finds all previously reported isomers (including global minima candidates) and additionally finds three new low energy isomers. New global minima candidate structures are generated for $\left(\mathrm{SiO}_{2}\right)_{M} \cdot\left(\mathrm{H}_{2} \mathrm{O}\right)_{N}$ systems with $M=6,10$ and 12 , each for a range of $\mathrm{N}$ values. The hydroxylation reaction energy trends for these series are consistent with previous works on other cluster sizes. The hydroxylation degree shows a threshold in each case when the hydroxylation-induced structural transformations minimises the tetrahedral distortion around the $\mathrm{SiO}_{4}$ centres. At this optimal hydroxylation $\left(R_{N / M}^{o p t}\right)$ point the reaction energy starts to level off to a constant value. Confirming previously reported results for other cluster sizes[18], we confirm that the size $(M)$ dependence of $R_{N / M}^{o p t}$ follows an inverse power law with an exponent close to $-2 / 3$.

\section{Acknowledgements}

Support from Spanish MINECO/FEDER grant CTQ2015-64618-R grant and, in part, by Generalitat de Catalunya grants 2014SGR97, XRQTC is acknowledged. We also acknowledge the NOMAD Center of Excellence project (this project has received funding from the European Union's Horizon 2020 research and innovation programme under grant agreement No 676580). Access to supercomputer resources was provided through grants from the Red Española de Supercomputación. We acknowledge funding from H2020 project ITN-EJD-642294 (TCCM: Theoretical Chemistry and Computational Modelling). We also thank Ask Hjorth Larsen for technical advice concerning the Atomic Simulation Environment.

\section{References}

[1] P. Aggarwal, R. Pratap Singh, Y. Aggarwal, Use of nano-silica in cement based materials - A review, Cogent Engineering 2 (2015) 1078018(1-11).

[2] S. Zhoua, L. Wu, J. Suna, W. Shen, The change of the properties of acrylic-based polyurethane via addition of nano-silica, Progress in Organic Coatings 45 (2002) 33-42.

[3] M. Qu, J. S. Meth, G. S. Blackman, G. M. Cohen, K. G. Sharp, K. J. Van Vliet, Tailoring and probing particlepolymer interactions in PMMA/silica nanocomposites, Soft Matter 7 (2011) 8401-8408.

[4] L. T. Zhuravlev, The surface chemistry of amorphous silica. Zhuravlev model, Colloids and Surfaces A: Physicochemical and Engineering Aspects 173 (2000) 1-38.

[5] C. S. Cundya, P. A. Cox, The hydrothermal synthesis of zeolites: Precursors, intermediates and reaction mechanism, Microporous and Mesoporous Materials 82 (2005) 1-78.

[6] D. J. Belton, O. Deschaume, C. C. Perry, An overview of the fundamentals of the chemistry of silica with relevance to biosilicification and technological advances, FEBS Journal 279 (2012) 1710-1720

[7] P. Chandra, D. S. Doke, S. B. Umbarkar, K. Vank, A. V. Biradar, Silica microspheres containing high density surface hydroxyl groups as efficient epoxidation catalysts, RSC Adv. 5 (2015) 21125-21131.

[8] Y. Wang, M. Y. He and R. Y. Chen, Fabrication of mechanically robust antireflective films using silica nanoparticles with enhanced surface hydroxyl groups, J. Mater. Chem. A 3 (2015) 1609-1618.

[9] A. Marucco, F. Turci, L. O'Neill, H. J. Byrne, B. Fubini, I. Fenoglio, Hydroxyl density affects the interaction of fibrinogen with silica nanoparticles at physiological concentration, Journal of Colloid and Interface Science 419 (2014) 86-94.

[10] H. Zhang, D. R. Dunphy, X. Jiang, H. Meng, B. Sun, D. Tarn, M. Xue, X. Wang, S. Lin, Z. Ji, R. Li, F. L. Garcia, J. Yang, M. L. Kirk, T. Xia, J. I. Zink, A. Nel, C. J. Brinker, Processing Pathway Dependence of Amorphous Silica Nanoparticle Toxicity: Colloidal vs Pyrolytic, J. Am. Chem. Soc. 134 (2012) 15790-15804.

[11] J. Yang, E.G. Wang, Reaction of water on silica surfaces, Current Opinion in Solid State and Materials Science 10 (2006) 33-39.

[12] M. J. Mora-Fonz, C. R. A. Catlow, D. W. Lewis, Oligomerization and Cyclization Processes in the Nucleation of Microporous Silicas, Angew. Chem. Int. Ed. 44 (2005) 3082 -3086. 
[13] T. T. Trinh, Antonius P. J. Jansen, R. A. van Santen, Mechanism of Oligomerization Reactions of Silica, J. Phys. Chem. B, 110 (2006) 23099-23106.

[14] D. Makimura, C. Metin, T. Kabashima, T. Matsuoka, Q. P. Nguyen, C. R. Miranda, Combined modeling and experimental studies of hydroxylated silica nanoparticles, J. Mater. Sci. 45 (2010) 5084-5088.

[15] J. Yeon, A.C.T. van Duin, ReaxFF Molecular Dynamics Simulations of Hydroxylation Kinetics for Amorphous and Nano-Silica Structure, and Its Relations with Atomic Strain Energy, J. Phys. Chem. C 120 (2016) 305-3017. [16] S. Bhattacharya, J. Kieffer, Molecular Dynamics Simulation Study of Growth Regimes during Polycondensation of Silicic Acid: from Silica Nanoparticles to Porous Gels, J. Phys. Chem. C 112 (2008) 17641771.

[17] S. T. Bromley, E. Flikkema, Novel structures and energy spectra of hydroxylated $\left(\mathrm{SiO}_{2}\right)_{8}$-based clusters: searching for the magic $\left(\mathrm{SiO}_{2}\right)_{8} \mathrm{O}_{2} \mathrm{H}_{3}$ cluster, J. Chem. Phys., 122, (2005) 114303/1-114303/5.

[18] K.E. Jelfs, E. Flikkema, S.T. Bromley, Hydroxylation of silica nanoclusters $\left(\mathrm{SiO}_{2}\right)_{M}\left(\mathrm{H}_{2} \mathrm{O}\right)_{N}, M=4,8,16,24$ : stability and structural trends, Phys. Chem. Chem. Phys. 15 (2013) 20438-20443.

[19] E. Flikkema, K.E. Jelfs, S. T. Bromley, Structure and energetics of hydroxylated silica clusters, $\left(\mathrm{SiO}_{2}\right)_{M}\left(\mathrm{H}_{2} \mathrm{O}\right)_{N}$, $M=8,16$ and $N=1-4$ : A global optimisation study, Chem. Phys. Lett. 554 (2012) 117-122.

[20] Evidence for atomic mixing via multiple intermediates during the dynamic interconversion of silicate oligomers in solution, K. E. Jelfs, E. Flikkema, S. T. Bromley, Chem. Comm. 48 (2012) 46-48.

[21] D. J. Wales, J. P. K. Doye, J. Phys. Chem. A 1997, 101, 5111.

[22] N. Metropolis, A.W. Rosenbluth, M.N. Rosenbluth. A.H. Teller and E. Teller, Equation of State Calculations by Fast Computing Machines, J. Chem. Phys. 21 (1953) 1087-1092.

[23] J.D. Gale, GULP: A computer program for the symmetry-adapted simulation of solids, J. Chem. Soc., Faraday Trans. 93 (1997) 629-637.

[24] S. R. Bahn, K. W. Jacobsen, An object-oriented scripting interface to a legacy electronic structure code, Comput. Sci. Eng. 4 (2002) 56-66.

[25] S. Tsuneyuki, M. Tsukada, H. Aoki, Y. Matsui, First-Principles Interatomic Potential of Silica Applied to Molecular Dynamics, Phys. Rev. Lett. 61 (1988) 869-872.

[26] B. van Beest, G. Kramer, R. van Santen, Force fields for silicas and aluminophosphates based on ab initio calculations, Phys. Rev. Lett. 64 (1990) 1955-1958.

[27] A. Hassanali, S. J. Singer, J. Phys. Chem. B 2007, 111, 11181.

[28] E. Flikkema, S.T. Bromley, A new interatomic potential for nanoscale silica, Chem. Phys. Lett. 378 (2003)

622-629.

[29] A. Pedone, G. Malavasi, M.C. Menziani, U. Segre, F. Musso, M. Corno, B. Civalleri, P. Ugliengo, FFSiOH: a New Force Field for Silica Polymorphs and Their Hydroxylated Surfaces Based on Periodic B3LYP Calculations, Chem. Mater. 20 (2008) 2522-2531.

[30] A. Maciá, S. T. Bromley, in preparation.

[31] V. Blum, R. Gehrke, F. Hanke, P. Havu, V. Havu, X. Ren, K. Reuter, M. Scheffler, Phys. Commun., 180 (2009) 2175-2196.

[32] A.D. Becke, Density-functional thermochemistry. III. The role of exact exchange, J. Phys. Chem. 98 (1993) 5648-5652.

[33] M. Delle Piane, M. Corno, A. Pedone, R. Dovesi, P. Ugliengo, Large-Scale B3LYP Simulations of Ibuprofen Adsorbed in MCM-41 Mesoporous Silica as Drug Delivery System, J. Phys. Chem. C 118 (2014) 26737-26749.

[34] A. Rimola, D. Costa, M. Sodupe, J. Lambert, P. Ugliengo, Silica Surface Features and Their Role in the Adsorption of Biomolecules: Computational Modeling and Experiments, Chem. Rev. 113 (2013) 4216-4313. [35] V.G. Avakyan, V.F. Sidorkin, E.F. Belogolova, S.L. Guselnikov, L.E. Guselnikov, AIM and ELF electronic structure/G2 and $\mathrm{G} 3 \pi$-bond energy relationship for doubly bonded silicon species, $\mathrm{H} 2 \mathrm{Si}=\mathrm{X}(\mathrm{X}=\mathrm{E} 14 \mathrm{H} 2, \mathrm{E} 15 \mathrm{H}$, E16), Organometallics 25 (2006) 6007-6013.

[36] M. A. Zwijnenburg, A. A. Sokol, C. Sousa, S. T. Bromley, The effect of local environment on photoluminescence: A time-dependent density functional theory study of silanone groups on the surface of silica nanostructures, J. Chem. Phys., 2009, 131, 034705.

[37] S. Hamad and S. T. Bromley, Low reactivity of non-bridging oxygen defects on stoichiometric silica surfaces, Chem. Commun. (2008) 4156-4158.

[38] M. A. Zwijnenburg, F. Illas, S. T. Bromley, Long range coupling between defect centres in inorganic nanostructures: Valence alternation pairs in nanoscale silica, J. Chem. Phys. 137 (2012) 154313

[39] S. T. Bromley, I. de P. R. Moreira, F. Illas, J. C. Wojdet, Importance of the embedding environment on the strain within small rings in siliceous materials, Phys. Rev. B 73 (2006) 134202. 
[40] R.L. Johnston, Atomic and Molecular Clusters, Taylor \& Francis, London and New York, 2002. 